\title{
Synchrotron-Infrared Microscopy Analysis of Amyloid Fibrils Irradiated by Mid-Infrared Free-Electron Laser
}

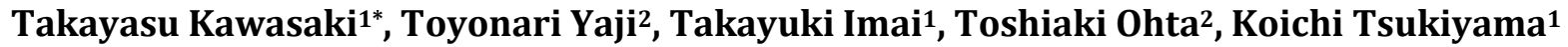 \\ ${ }^{1}$ IR Free Electron Laser Research Center, Research Institute for Science and Technology (RIST), \\ Tokyo University of Science, Noda, Japan \\ ${ }^{2}$ SR Center, Research Organization of Science and Engineering, Ritsumeikan University, Kusatsu, Japan \\ Email: ${ }^{*}$ kawasaki@rs.tus.ac.jp
}

Received 24 February 2014; revised 27 March 2014; accepted 5 April 2014

Copyright (C) 2014 by authors and Scientific Research Publishing Inc.

This work is licensed under the Creative Commons Attribution International License (CC BY). http://creativecommons.org/licenses/by/4.0/

(c) (i) Open Access

\section{Abstract}

Amyloid fibrils are widely recognized as a cause of serious amyloidosis such as Alzheimer's disease. Although dissociation of amyloid fibril aggregates is expected to lead to a decrease in the toxicity of the fibrils in cells, the fibril structure is robust under physiological conditions. We have irradiated amyloid fibrils with a free-electron laser (FEL) tuned to mid-infrared frequencies to induce dissociation of the aggregates into monomer forms. We have previously succeeded in dissociating fibril structures of a short peptide of the thyroid hormone by tuning the oscillation frequency to the amide I band, but the detailed structural changes of the peptide have not yet been determined at a high spatial resolution. Synchrotron-radiation infrared microscopy (SR-IRM) is a powerful tool for in situ analysis of minute structural changes of various materials, and in this study, the feasibility of SR-IRM for analyzing the microscopic conformational changes of amyloid fibrils after FEL irradiation was investigated. Reflection spectra of the amyloid fibril surface showed that the amide I peaks shifted to higher wave numbers after the FEL irradiation, indicating that the initial $\beta$-sheet-rich structure transformed into a mixture of non-ordered and turn-like peptide conformations. This result demonstrates that conformational changes of the fibril structure after the FEL irradiation can be observed at a high spatial resolution using SR-IRM analysis and the FEL irradiation system can be useful for dissociation of amyloid aggregates.

\section{Keywords}

Amyloid Fibrils, Free-Electron Laser, Infrared Microscopy, Synchrotron Radiation

\footnotetext{
${ }^{*}$ Corresponding author.
} 


\section{Introduction}

Amyloidosis is caused by the deposition of amyloid fibrils in various organs of a mammalian body [1]-[3]. A common form of amyloidosis, Alzheimer's disease, is becoming more common as the population increases, and Alzheimer Disease International (ADI) has estimated that there will be ca.100 million Alzheimer's disease patients worldwide in 2050 [4]. Furthermore, cancer-associated diseases such as multiple myeloma are caused by amyloidosis, and an effective therapy for the amyloidosis has not yet been developed [5]. Amyloid fibrils are recognized as a clinical target, and they have a common secondary structure, cross- $\beta$ structure, that is formed by peptides and various proteins [3]. Decreasing the amount of amyloid fibrils in tissues is considered to be an effective treatment for amyloidosis, but it is difficult to dissociate the robust fibril structure unless organic solvents or synthetic molecules are used [6] [7].

We have recently found that a free-electron laser (FEL) tuned to the amide I band $\left(1600-1700 \mathrm{~cm}^{-1}\right)$ is able to dissociate the amyloid fibrils of lysozyme and of a five-residue peptide (DFNKF) of the thyroid hormone [8] [9]. In the case of lysozyme fibrils, the $\beta$-sheet content of the fibrils diminishes, and the enzyme can be refolded into the active form after the FEL irradiation [8]. FEL can deliver picosecond pulses, high-photon density, and high-power radiation [10]-[14], and it can be suggested that non-covalent bonds such as hydrogen bonds between the $\beta$-sheet structures of amyloid fibrils are cleaved by the high-powered pulsed laser tuned to the frequency of amide $\mathrm{C}=\mathrm{O}$ stretching vibration to induce the disaggregation of amyloid fibrils [8]. The structural change was determined by using conventional Fourier transform infrared (FTIR) spectroscopy in the previous study. In the case of the short peptide, however, FTIR spectroscopy could not determine the conformational changes in detail because the peptide has several conformations and a flexible structure in solution [9]. X-ray crystallography and nuclear magnetic resonance (NMR) are usually employed to determine the structure of peptides [15]-[17], but while these analytical methods are excellent for three-dimensional structural determination at the atomic level, they are also time consuming, and once analyzed, samples cannot be re-used for other analytical methods. In contrast, IR absorption spectral measurements are comparatively simple, and the spectra are sensitive to the secondary structures of the peptides [18]. Moreover, the normal structure of a peptide can be easily distinguished from the amyloid fibril structure in IR spectra because a peak of the amide I band shifts to as smaller wavenumber as the content of $\beta$-sheet structure increases during fibrillation of the peptide [19] [20]; IR absorption measurements are therefore also useful for detecting amyloid fibrils, as reviewed in a recent paper [21]. IR microscopy analysis is often employed for studying the local structure of organic materials and biomaterials [22] [23], and its advantages lie in the fact that no labeling or pre-treatment of the sample is required, which means samples can be re-used. The use of synchrotron radiation in combination with IR microscopy analysis improves the spatial resolution with a high signal-to-noise $(\mathrm{S} / \mathrm{N})$ ratio compared to that using a thermal radiation beam because high-power light can be delivered to a small area in a small sample (of several micrometers section) [24]. Synchrotron radiation IR microscopy (SR-IRM) has recently been used for monitoring the protein secondary structures of silk and investigating protein phosphorylation in living cells [25] [26]. Encouraged by these studies, we investigated the use of SR-IRM for detecting the minute (conformational) structural changes of amyloid fibrils formed by a short peptide after irradiation with an FEL tuned to the amide I band.

\section{Materials and Methods}

\subsection{Materials}

All of the reagents used in this study were purchased as special-grade chemicals. Tris-base, dimethyl sulfoxide (DMSO), hydrochloric acid, phosphate buffered saline (PBS; $10 \mathrm{mM}$ ), and sodium chloride were purchased from Wako Pure Chemical Industries (Osaka, Japan); 1,1,1,3,3,3-hexafluoro-2-propanol (HFP) from SigmaAldrich (St. Louis, USA); synthesized pentapeptide, DFNKF (80.9\% purity), from PH Japan Co., Ltd. (Hiroshima, Japan); and A $\beta 42$ (90\% purity) from Peptide Institute Inc. (Osaka, Japan).

\subsection{Preparation of Amyloid Fibrils and Irradiation with the FEL}

The thyroid hormone pentapeptide, DFNKF, was dissolved in 10\% DMSO in buffer A, which contained $10 \mathrm{mM}$ of tris-base and $20 \mathrm{mM}$ of $\mathrm{NaCl}$; the $\mathrm{pH}$ of the solution was adjusted to 7.5 using $\mathrm{HCl}$. The peptide powder was dissolved at a concentration of $100 \mathrm{mg} / \mathrm{mL}$ in DMSO and then diluted to $10 \mathrm{mg} / \mathrm{mL}$ using buffer A. The solution 
was incubated for two days at $37^{\circ} \mathrm{C}$, after which $100 \mu \mathrm{L}$ of the solution containing amyloid fibrils was placed on a stainless-steel substrate (Jasco International Co., Ltd., Tokyo, Japan) and irradiated with the FEL beam (tuned to the amide I band) at $37^{\circ} \mathrm{C}$. To prevent water evaporation, $10 \mu \mathrm{L}$ of water was added periodically to the suspension during irradiation. After irradiation, the sample on the substrate was air-dried before IR absorption measurements were performed. The $\mathrm{A} \beta 42$ was dissolved in $0.27 \mathrm{~mL}$ of HFP and dried under reduced pressure. The dried pellet was then re-dissolved in DMSO at $1.0 \mathrm{mM}$ as a stock solution, and the $\mathrm{A} \beta$ solution was diluted with PBS to $0.1 \mathrm{mM}$ and incubated at $37^{\circ} \mathrm{C}$ for $24 \mathrm{~h}$.

\subsection{Mid-Infrared Free-Electron Laser at the Tokyo University of Science (FEL-TUS)}

The FEL-TUS uses synchrotron radiation (SR) as a seed to generate a laser beam with a variable wavelength in the mid-infrared region (5.0 - $16 \mu \mathrm{m} ; 625-2000 \mathrm{~cm}^{-1}$ ) (Figure 1) [14]. An electron beam generated by a highradio-frequency (RF) electron gun $(2856 \mathrm{MHz})$ is accelerated to a maximum energy of $40 \mathrm{MeV}$ by a linear accelerator and injected into an undulator (a periodic magnetic field). The oscillations of the electron beam in the undulator generate SR. The maximum value of the periodic magnetic flux density is set to $0.83 \mathrm{~T}$, and the amplified SR is reflected upstream of the electron beam by a mirror positioned downstream of the beam, and then re-reflected by the upstream mirror to interact with the electron beam again, producing coherent laser light. The FEL-TUS provides two types of laser pulses: macro- and micropulses. Macropulses have a duration of $\sim 2 \mu \mathrm{s}$ and a repetition rate of $5 \mathrm{~Hz}$ during operation; the macropulses consists of a train of 2-ps micropulses separated by an interval of $350 \mathrm{ps}$. The energy of each laser macropulse used for the current experiment was in the range of 6.0 - $8.0 \mathrm{~mJ}$, as measured using an energy meter (SOLO2, Gentec-EO Inc., Quebec, Canada). Prior to the irradiation, the beam was focused to a point above the sample using a He-Ne beam. The spot size of beam line was ca. $0.5 \mathrm{~cm}$ in diameter.

\subsection{Synchrotron Radiation Infrared (SR-IR) Microscopy}

The SR-IR microscopic analysis was performed using the IR micro-spectroscopy beamline (SRMS, BL-15) at the SR center of Ritsumeikan University [27]. The beamline is equipped with Nicolet 6700 and Continuum XL

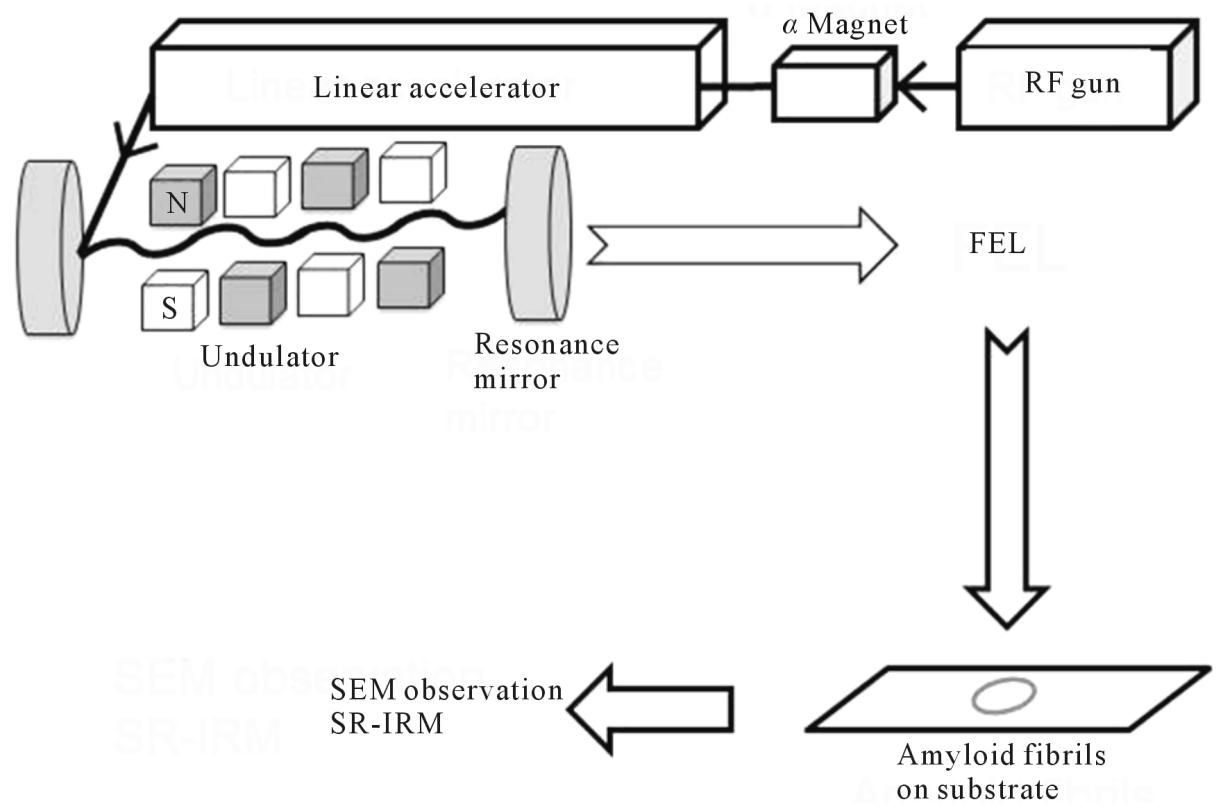

Figure 1. Schematic of the FEL and analysis setup. The laser beam is generated using an electronic gun and accelerated to $40 \mathrm{MeV}$ by the linear accelerator. The periodic length of the undulator can be tuned to set the oscillation wavelength. The FEL beam is transported to the laboratory though a vacuum tube, and the irradiation to the sample on the subatrate is controlled by a gold-coated mirror. After irradiation, the sample was analyzed using scanning electron microscopy (SEM) and SR-IRM. 
IR microscopes (Thermo Fisher Scientific Inc.). Measurements were performed in reflection mode with a $32 \times$ Cassegrain lens and a $10 \mu \mathrm{m} \times 10 \mu \mathrm{m}$ aperture. Spectra were collected in the mid-IR range of $700-4000 \mathrm{~cm}^{-1}$ at a resolution of $4 \mathrm{~cm}^{-1}$ with 256 scans. Smoothing and normalization of spectra were performed on the amide I band $\left(1600-1700 \mathrm{~cm}^{-1}\right.$ ) by using Spectra Manager software Ver. 2 (Jasco International Co., Ltd., Tokyo, Japan).

\subsection{Scanning Electron Microscopy}

The morphologies of the DFNKF amyloid fibrils were analyzed using a Supra40 field-emission scanning electron microscope (FE-SEM; Carl Zeiss). The SEM samples were prepared by placing $100 \mu \mathrm{L}$ of the fibril solution on a glass slide, which was then air-dried and fixed to the sample holder using conductive copper tape. The acceleration voltage was set to $7.00 \mathrm{kV}$.

\section{Results}

\subsection{Effect of Free-Electron Laser Irradiation on the Dissociation of Peptide Amyloid Fibrils}

In our previous study, we found that FEL irradiation of DFNKF amyloid fibrils caused disaggregation when the FEL light was tuned to the amide I band $\left(6.08 \mu \mathrm{m}, c a .1644 \mathrm{~cm}^{-1}\right)$ [9]. Since the peak intensity of the peptide increased after the fibrillation compared to the other peaks within amide I region $\left(1600-1700 \mathrm{~cm}^{-1}\right)$, we considered that the increase of the peak intensity reflected the $\beta$-sheet-rich fibril structure of the peptide. After the FEL irradiation tuned to $6.08 \mu \mathrm{m}$, we confirmed the effect of FEL on the disaggregation of the peptide fibrils by using SEM. Figure 2 shows SEM images of the amyloid fibrils before and after FEL irradiation. The SEM image of the amyloid fibrils before irradiation reveals that there were a number of fibril bundles approximately 100 $\mu \mathrm{m}$ long and $10 \mu \mathrm{m}$ wide connected in the form of a net (Figure 2(a)). Each bundle contains two or three fibrils that are several micrometers wide. After irradiation, however, there appeared to be fewer fibrils, and several globular solids about $10 \mu \mathrm{m}$ in diameter were seen (Figure 2(b)). This result is consistent with the previous results demonstrating that FEL light tuned to the amide I band will dissociate amyloid fibrils [8] [9]. It can be considered that the FEL irradiation at the amide I band heats the fibrils and the surrounding water, driving the dissociation of the fibrils. Under the dissociation mechanism of amyloid fibrils, it can be suggested that noncovalent bonds such as hydrogen bonds between $\beta$-sheet structures of amyloid fibrils can be affected by the FEL during the irradiation process. Since the specificity of the FEL frequency for the dissociation of amyloid fibrils was shown in the previous study, only the amide I frequency was used in this study.

\subsection{Analysis by Synchrotron-Radiation Infrared Microscopy (SR-IRM)}

The surface area of the amyloid fibrils was analyzed using SR-IRM at several positions with $10 \times 10 \mu \mathrm{m}$ square aperture (Figure 3 and Figure 4). Since the minimum size of fibrils bundle was estimated to be around $10 \mu \mathrm{m}$ as shown in the SEM image (Figure 2), this aperture size was selected in this study. Spectra were collected from areas with a lighter contrast in the microscope image to suppress the background contribution in reflection mode (Figure 3(a) and Figure 4(a)).The positions for measurement were numbered in those images. A total of nine spectra were collected from the samples both before and after FEL irradiation over a wavenumber range of 1600 $1700 \mathrm{~cm}^{-1}$ (the amide I region). Almost of reflection spectra indicated that before FEL irradiation the amyloid fibrils had two major absorption bands: $<1640 \mathrm{~cm}^{-1}$ and around $1670 \mathrm{~cm}^{-1}$ (Figure 3(b) and Figure 3(c)). The former bands were ranged from $1635 \mathrm{~cm}^{-1}$ to $1640 \mathrm{~cm}^{-1}$, and the latter bands were from $1668 \mathrm{~cm}^{-1}$ to $1670 \mathrm{~cm}^{-1}$. The spectrum pattern having two major amide I bands is characteristic of the short peptide as indicated in the previous study [9] and distinct from that of large protein such as lysozyme which has single major amide I band [8].This means that there are several conformations in the fibrils structure of the DFNKF peptide, that is antiparallel $\beta$-sheet structures mixed with non-ordered structures and turns [21] [28] [29]. Although a possibility that the latter bands correspond to the amide side chain of asparagine $(\mathrm{N})$ cannot be excluded, the absorbance of the amide backbone is probably strong in contrast to that of the side chain. These spectra imply that the almost the whole sample surface was covered with amyloid fibrils, although spectra from the entire area were not collected. On the contrary, the peaks in the SR-IR spectra around 1640 and $1670 \mathrm{~cm}^{-1}$ shifted to larger wavenumb- 


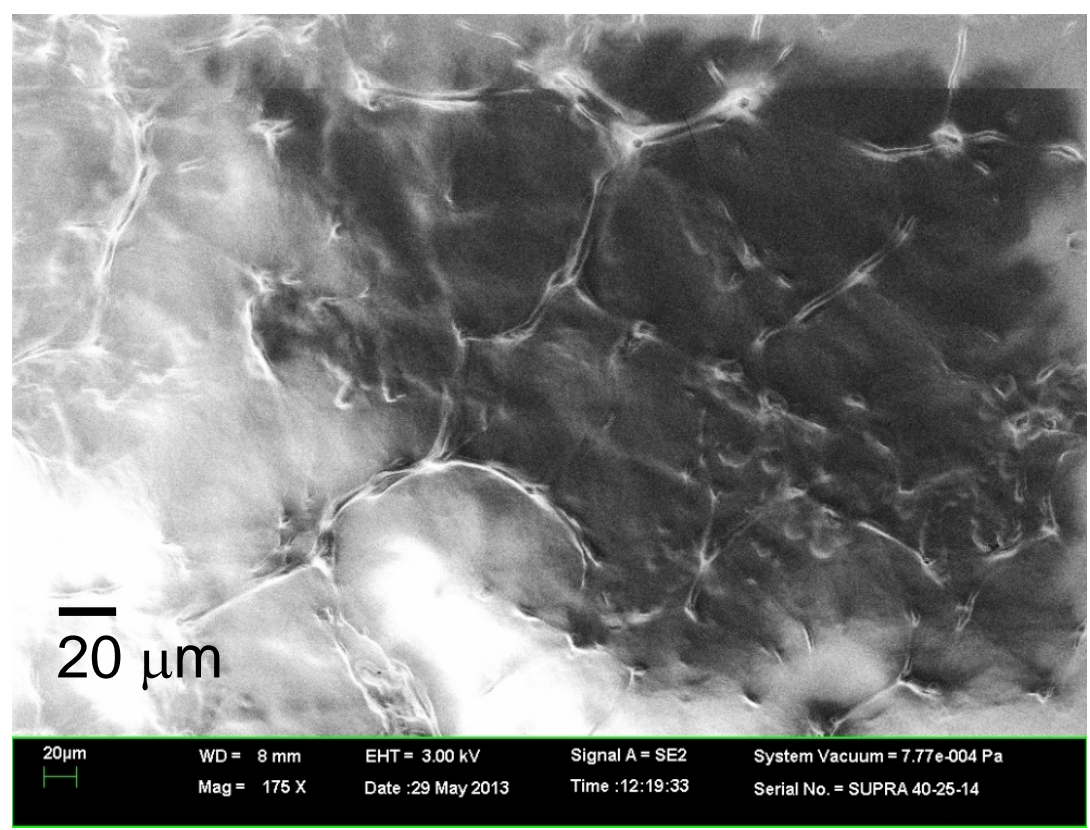

(a)

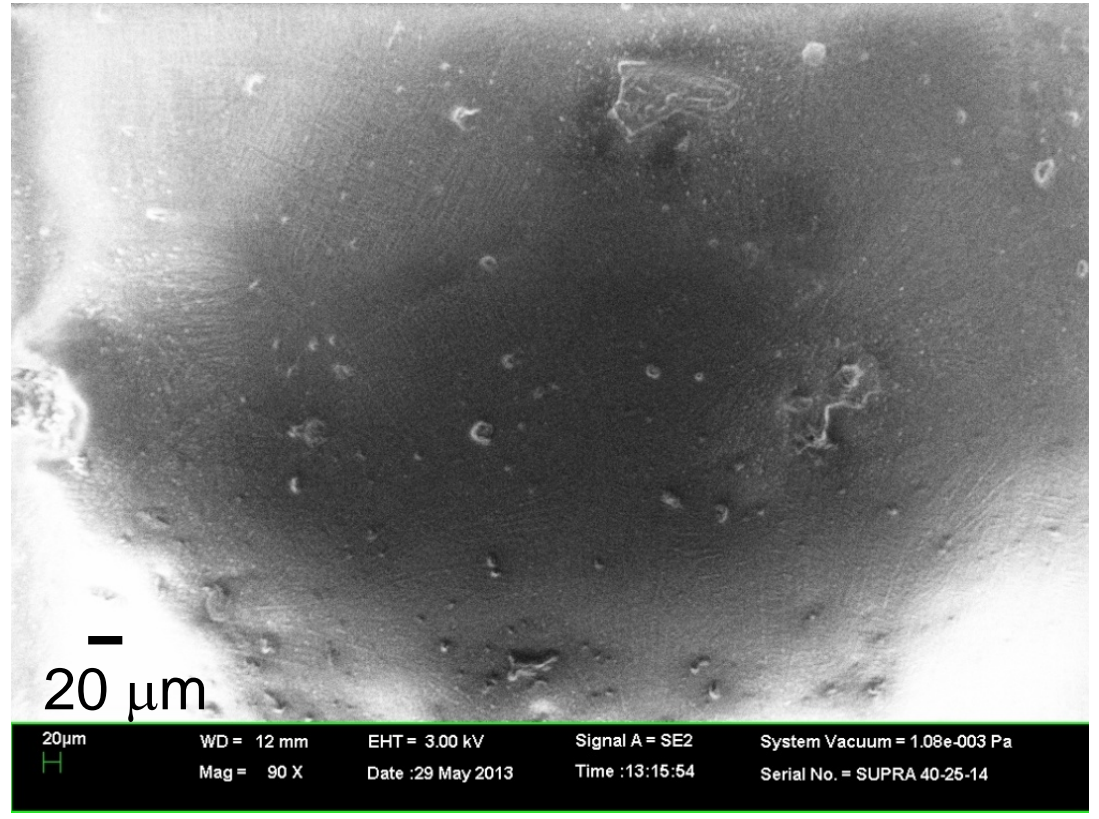

(b)

Figure 2. SEM image of the amyloid fibrils (a) before and (b) after FEL irradiation. Images are shown at a magnification of 175.

ers after FEL irradiation (Figure 4(b) and Figure 4(c)). Some spectra (no.1 and no.4) exhibited peaks close to $1680 \mathrm{~cm}^{-1}$. Moreover, both bands around 1640 and $1670 \mathrm{~cm}^{-1}$ had a width of about $30 \mathrm{~cm}^{-1}$ after the irradiation, while those had a width of about $20 \mathrm{~cm}^{-1}$ before the irradiation, that is the widths of amide I bands after the FEL irradiation were broad compared to those before the irradiation. These results indicate that there were fewer $\beta$-sheet structures and more non-ordered and turns structures in the local fibrils after FEL irradiation [21] [29]. Although more concrete secondary conformations of peptides could not be determined by these analyses, the above results clearly indicate that the FEL irradiation is capable of disaggregating peptide amyloid fibrils and can produce substantially non- $\beta$-sheet peptides. This is consistent with the previous study indicating that the 


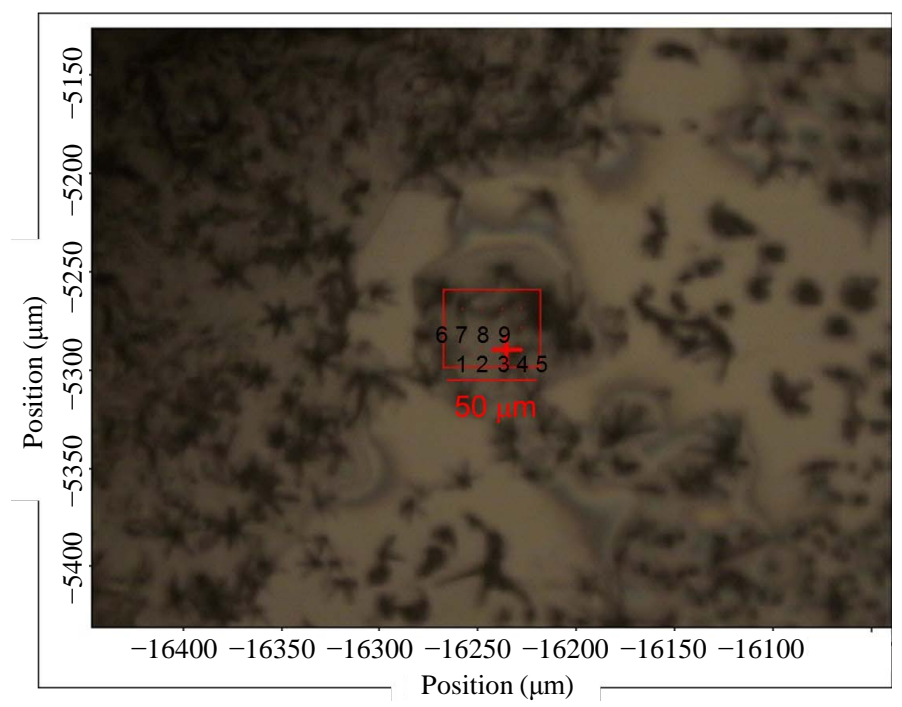

(a)

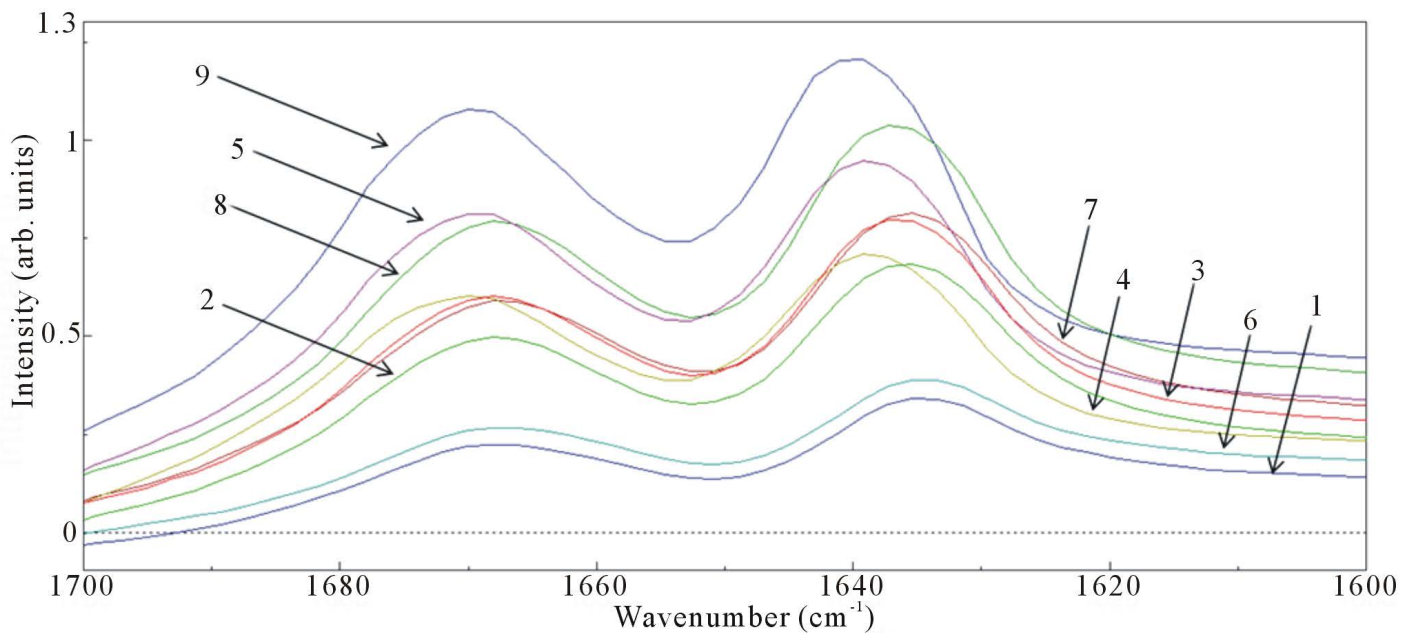

(b)

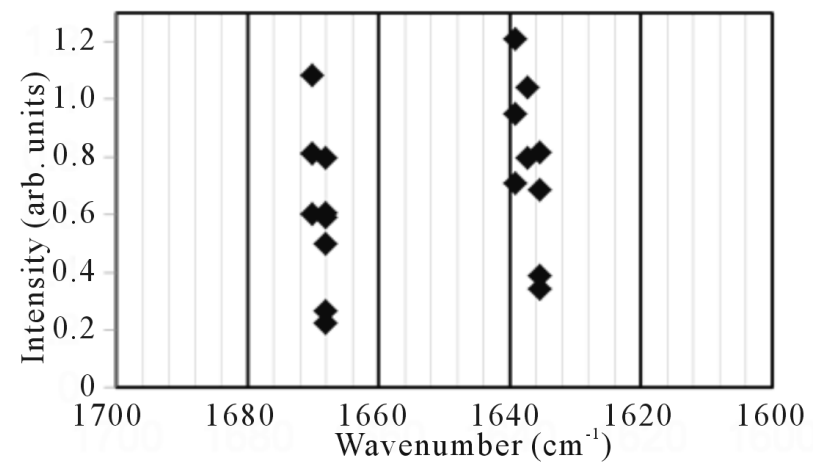

(c)

Figure 3. (a) SR-IRM image of the DFNKF amyloid fibrils before FEL irradiation. The target size was 50 $\mu \mathrm{m} \times 40 \mu \mathrm{m}$. The numbers (no.1 - 9) indicate the positions for measurements of spectra; (b) IR reflection spectra; (c) Plot of the peaks in the amide I region.

FEL tuned to the amide I band could dissociate the amyloid aggregates of lysozyme and the short peptide [8] [9]. For comparison, we also measured SR-IR spectra of $\mathrm{A} \beta$ fibrils, formed with $10 \mu \mathrm{g}\left(2.5 \times 10^{-9} \mathrm{~mol}\right)$ of $\mathrm{A} \beta 42$ 


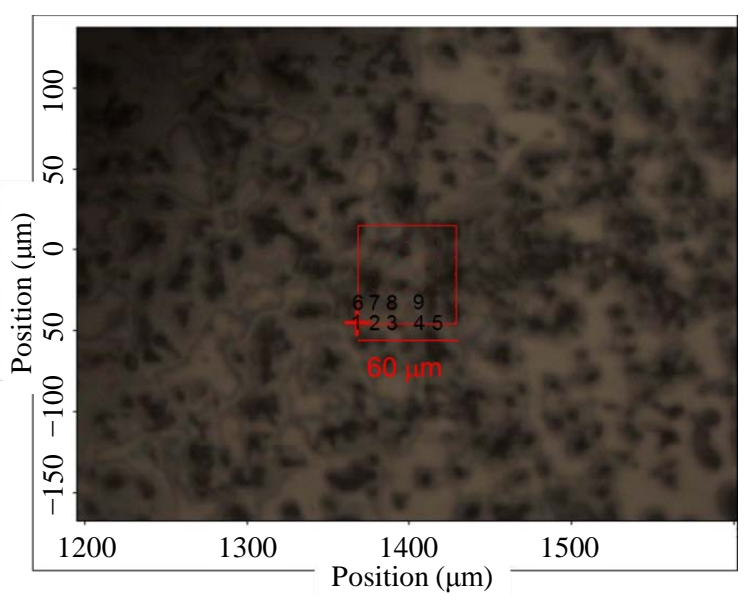

(a)

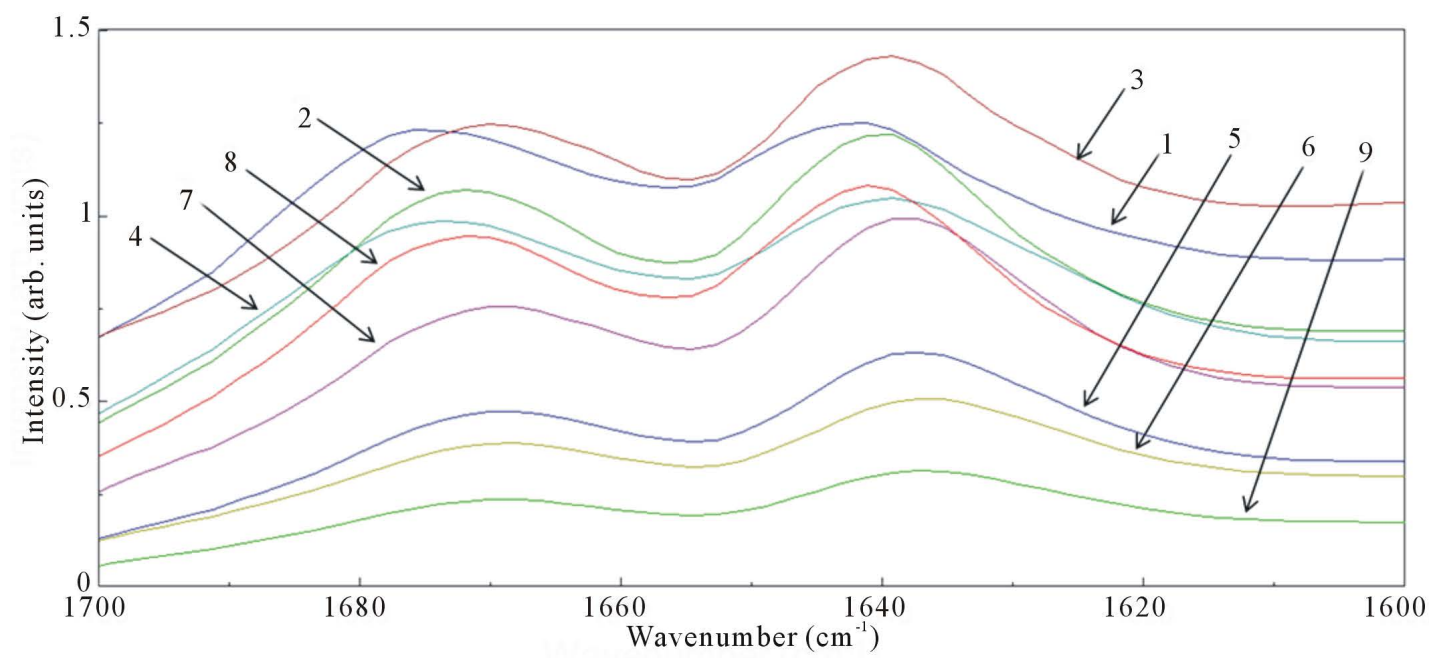

(b)

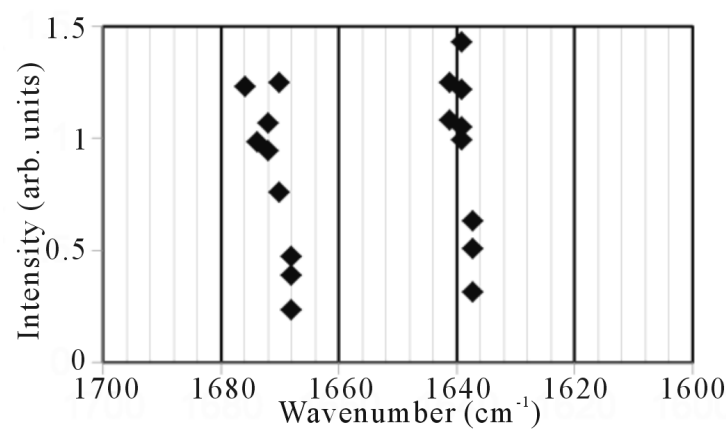

(c)

Figure 4. (a) SR-IRM image of the DFNKF amyloid fibrils after FEL irradiation. The target size was $60 \mu \mathrm{m} \times$ $60 \mu \mathrm{m}$. The numbers (no.1 - 9) indicate the positions for measurements of spectra; (b) IR reflection spectra; (c) Plot of the peaks in the amide I region.

peptide using the same method (Figure 5(a) and Figure 5(b)). The sample amount was small; nevertheless the reflection spectra of the $\mathrm{A} \beta$ fibrils could be obtained with high $\mathrm{S} / \mathrm{N}$ ratio and exhibited a main absorption peak below $1630 \mathrm{~cm}^{-1}$, which is associated with a typical $\beta$-sheet-rich structure [21]. From a comparison of the IR spectra of the DFNKF and $\mathrm{A} \beta$ fibrils we can conclude that there is a conformational difference in the secondary structure, although both peptides form similar fibril structures. In particular, there is a number of peptide con- 


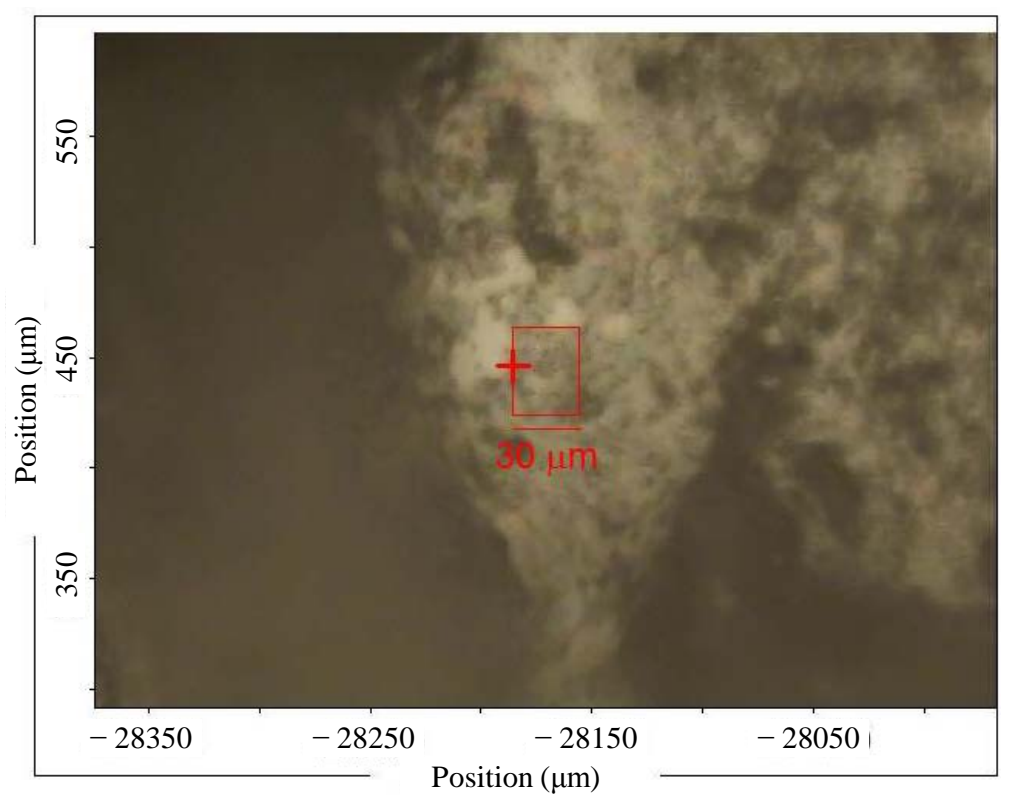

(a)

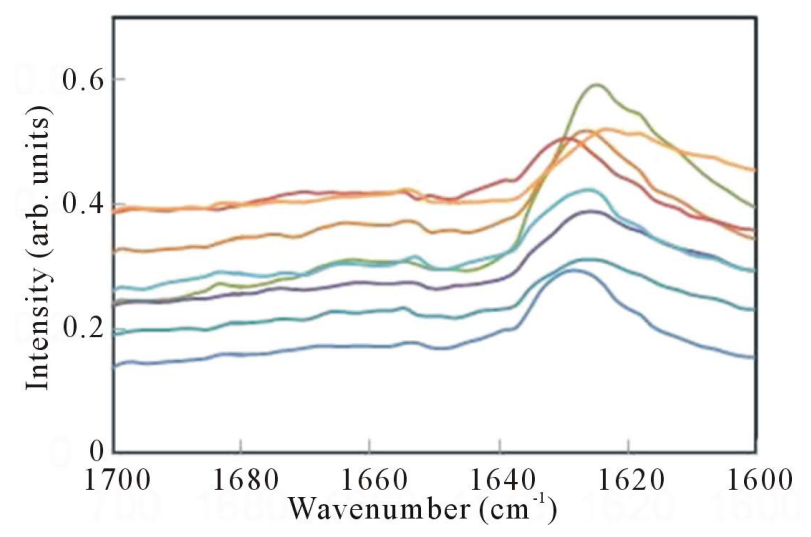

(b)

Figure 5. (a) SR-IRM image of the A $\beta 42$ amyloid fibrils. The target size was $30 \mu \mathrm{m} \times 40 \mu \mathrm{m}$; (b) IR reflection spectra.

formers in both amyloid fibrils, since the frequencies of the amide I peaks are different in both cases. Moreover, it can be considered that several-type oligomers of $\mathrm{A} \beta 42$ are included in the amyloid fibrils, because amide I peaks are varied from $1620 \mathrm{~cm}^{-1}$ to $1640 \mathrm{~cm}^{-1}$ (Figure 5(b)).

\section{Discussion}

In our previous study, we measured the FTIR spectra of amyloid fibrils from lysozyme and DFNKF peptide after they had been mixed with $\mathrm{KBr}$ powder [8] [9]. This meant that the sample could not be re-used, and we were unable to obtain any spatial information about the structural changes of the sample; however, the average structure influenced the spectrum. IR microscopy, in contrast, can reveal spectral information about the structural changes of the peptides without the need for labeling, and SR-IRM provides this information at a high spatial resolution. For a pathological diagnosis of amyloidosis, it is necessary to be able to detect local amyloid-fibril deposition areas in tissues, and the result of this study indicates that there is a possibility that SR-IRM could be used for this purpose [21]. Moreover, the implication that there are various peptide conformations within a small area of amyloid fibrils after FEL irradiation suggests that FEL irradiation may be an effective tool for dissociating aggregated amyloid structures. The SR-IRM system can be regarded as suitable for detecting a small amount 
of amyloid fibrils in pathological tissues because the results demonstrated that fibrils formed from small amount $(10 \mu \mathrm{g})$ of $\mathrm{A} \beta(2.5 \mathrm{nmol})$ could be spectrally observed with this system. It can be considered that this analytical system is an alternative to the conventional staining method using Congo-red reagent [1]. Furthermore, the fact that the structural changes of the amyloid fibrils after FEL irradiation could be observed using SR-IRM means that this analytical system can be considered as a possible screening assay for inhibitors targeting amyloid fibrils [7]. For example, the inhibitory effect of candidate drugs on the dissociation of amyloid fibrils on the substrate could be tested using SR-IRM as shown in this study.

This study has revealed that structural changes could be observed after FEL irradiation, but planned future work will focus on observing the changes in real time during FEL irradiation by connecting the IR microscope to the FEL beamline. Such a strategy is likely to allow an investigation of the structural machinery and folding kinetics of amyloid fibrils on a microscopic level, as described in a recent paper [30]. By using the FEL irradiation system combined with the IR microscopy, it can be expected that the disaggregation mechanism of amyloid fibrils will be investigated.

\section{Conclusion}

In conclusion, an FEL tuned to the amide I band was employed to irradiate peptide amyloid fibrils, and the structural changes of the fibrils were analyzed by using SR-IRM. The results demonstrated that rigid fibril structures disaggregate under irradiation by an FEL beam and are converted to flexible peptide conformations. The FEL irradiation system will become an effective tool for dissociation of pathological amyloid aggregates.

\section{Acknowledgements}

We thank Mr. Tetsuo Morotomi and Mr. Keiichi Hisazumi (Mitsubishi Electric System \& Service Co., Ltd.) for operating the FEL instrument and Mr. Jun Fujioka for his assistance in obtaining the microscope images. This work was supported in part by the Open Advanced Research Facilities Initiative and Photon Beam Platform Project of the Ministry of Education, Culture, Sport, Science and Technology, Japan.

\section{References}

[1] Woldemeskel, M. (2012) A Concise Review of Amyloidosis in Animals. Veterinary Medicine International, 2012, Article ID: 427296. http://dx.doi.org/10.1155/2012/427296

[2] Gertz, M.A. (2013) Immunoglobulin Light Chain Amyloidosis: 2013 Update on Diagnosis, Prognosis, and Treatment. American Journal of Hematology, 88, 417-425. http://dx.doi.org/10.1002/ajh.23400

[3] Dobson, C.M. (2001) The Structural Basis of Protein Folding and Its Links with Human Disease. Philosophical Transactions of the Royal Society B, 356, 133-145. http://dx.doi.org/10.1098/rstb.2000.0758

[4] Prince, M., Prina, M. and Guerchet, M. (2013) The World Alzheimer Report 2013 in the Global Voice on Dementia. Alzheimer Disease International. http://www.alz.co.uk/adi-research

[5] Petruzziello, F., Zeppa, P., Catalano, L., Cozzolino, I., Gargiulo, G., Musto, P., D’Auria, F., Liso, V., Rizzi, R., Caruso, N., Califano, C., Piro, E., Musso, M., Bonanno, V., Falcone, A.P., Tafuto, S., Raimondo, F.D., Laurentiis, M.D., Pane, F., Palombini, L. and Rotoli, B. (2010) Amyloid in Bone Marrow Smears of Patients Affected by Multiple Myeloma. Annals of Hematology, 89, 469-474. http://dx.doi.org/10.1007/s00277-009-0857-9

[6] Booth, D.R., Sunde, M., Bellotti, V., Robinson, C.V., Hutchinson, W.L., Fraser, P.E., Hawkins, P.N., Dobson, C.M., Radford, S.E., Blake, C.C.F. and Pepys, M.B. (1997) Instability, Unfolding and Aggregation of Human Lysozyme Variants Underlying Amyloid Fibrillogenesis. Nature, 385, 787-793.

[7] Cheng, B., Gong, H., Xiao, H., Petersen, R.B., Zheng, L. and Huang, K. (2013) Inhibiting Toxic Aggregation of Amyloidogenic Proteins: A Therapeutic Strategy for Protein Misfolding Diseases. Biochimica et Biophysica Acta, 1830, 4860-4871. http://dx.doi.org/10.1016/j.bbagen.2013.06.029

[8] Kawasaki, T., Fujioka, J., Imai, T. and Tsukiyama, K. (2012) Effect of Mid-Infrared Free-Electron Laser Irradiation on Refolding of Amyloid-Like Fibrils of Lysozyme into Native Form. The Protein Journal, 31, 710-716. http://dx.doi.org/10.1007/s10930-012-9452-3

[9] Kawasaki, T., Imai, T. and Tsukiyama, K. (2014) Use of a Mid-Infrared Free-Electron Laser (MIR-FEL) for Dissociation of the Amyloid Fibril Aggregates of a Peptide. Journal of Analytical Sciences, Methods and Instrumentation, 4, 9-18. http://dx.doi.org./10.4236/jasmi.2014.41002

[10] Edwards, G., Logan, R., Copeland, M., Reinisch, L., Davidson, J., Johnson, B., Maciunas, R., Mendenhall, M., Ossoff, 
R., Tribble, J., Werkhaven, J. and O’Day, D. (1994) Tissue Ablation by a Free-Electron Laser Tuned to the Amide II Band. Nature, 371, 416-419.

[11] Austin, R.H., Xie, A., van der Meer, L., Redlich, B., Lindgård, P.-A., Frauenfelder, H. and Fu, D. (2005) Picosecond Thermometer in the Amide I Band of Myoglobin. Physical Review Letters, 94, 128101.

http://dx.doi.org/10.1103/PhysRevLett.94.128101

[12] Xiao, Y., Guo, M., Zhang, P., Shanmugam, G., Polavarapu, P.L. and Hutson, M.S. (2008) Wavelength-Dependent Conformational Changes in Collagen after Mid-Infrared Laser Ablation of Cornea. Biophysical Journal, 94, 1359-1366. http://dx.doi.org/10.1529/biophysj.107.114389

[13] Oomens, J., Polfer, N., Moore, D.T., van der Meer, L., Marshall, A.G., Eyler, J.R., Meijer, G. and von Helden, G. (2005) Charge-State Resolved Mid-Infrared Spectroscopy of a Gas-Phase Protein. Physical Chemistry Chemical Physics, 7, 1345-1348. http://dx.doi.org/10.1039/B502322J

[14] Nomaru, K., Kawai, M., Yokoyama, M., Oda, F., Nakayama, A., Koike, H. and Kuroda, H. (2000) Optical Beam Transport System at FEL-SUT. Nuclear Instruments and Methods in Physics Research Section A, 445, 379-383.

[15] Apostol, M.I., Perry, K. and Surewicz, W.K. (2013) Crystal Structure of a Human Prion Protein Fragment Reveals a Motif for Oligomer Formation. Journal of the American Chemical Society, 135, 10202-10205. http://dx.doi.org/10.1021/ja403001q

[16] Paravastu, A.K., Leapman, R.D., Yau, W.-M. and Tycko, R. (2008) Molecular Structural Basis for Polymorphism in Alzheimer's $\beta$-Amyloid Fibrils. Proceedings of the National Academy of Sciences of the United States of America, 105, 18349-18354. http://dx.doi.org/10.1073/pnas.0806270105

[17] Itoh-Watanabe, H., Kamihira-Ishijima, M., Javkhlantugs, N., Inoue, R., Itoh, Y., Endo, H., Tuzi, S., Saitô, H., Ueda, K. and Naito, A. (2013) Role of Aromatic Residues in Amyloid Fibril Formation of Human Calcitonin by Solid-State ${ }^{13} \mathrm{C}$ NMR and Molecular Dynamics Simulation. Physical Chemistry Chemical Physics, 15, 8890-8901. http://dx.doi.org/10.1039/c3cp44544e

[18] Bandekar, J. (1992) Amide Modes and Protein Conformation. Biochimica et Biophysica Acta-Protein Structure and Molecular Enzymology, 1120, 123-143.

[19] Ahmad, A., Uversky, V.N., Hong, D. and Fink, A.L. (2005) Early Events in the Fibrillation of Monomeric Insulin. The Journal of Biological Chemistry, 280, 42669-42675. http://dx.doi.org/10.1074/jbc.M504298200

[20] Zandomeneghi, G., Krebs, M.R.H., McCammon, M.G. and Fändrich, M. (2004) FTIR Reveals Structural Differences between Native $\beta$-Sheet Proteins and Amyloid Fibrils. Protein Science, 13, 3314-3321. http://dx.doi.org/10.1110/ps.041024904

[21] Miller, L.M., Bourassa, M.W. and Smith, R.J. (2013) FTIR Spectroscopic Imaging of Protein Aggregation in Living Cells. Biochimica et Biophysica Acta, 1828, 2339-2346. http://dx.doi.org/10.1016/j.bbamem.2013.01.014

[22] Acerbo, A.S., Lawrence Carr, G., Judex, S. and Miller, L.M. (2012) Imaging the Material Properties of Bone Specimens Using Reflection-Based Infrared Microspectroscopy. Analytical Chemistry, 84, 3607-3613. http://dx.doi.org/10.1021/ac203375d

[23] Gautam, R., Chandrasekar, B., Deobagkar-Lele, M., Rakshit, S., Vinay Kumar, B.N., Umapathy, S. and Nandi, D. (2012) Identification of Early Biomarkers during Acetaminophen-Induced Hepatotoxicity by Fourier Transform Infrared Microspectroscopy. PLoS ONE, 7, Article ID: e45521. http://dx.doi.org/10.1371/journal.pone.0045521

[24] Kakoulli, I., Prikhodko, S.V., Fischer, C., Cilluffo, M., Uribe, M., Bechtel, H.A., Fakra, S.C. and Marcus, M.A. (2013) Distribution and Chemical Speciation of Arsenic in Ancient Human Hair Using Synchrotron Radiation. Analytical Chemistry, 86, 521-526. http://dx.doi.org/10.1021/ac4024439

[25] Ling, S., Qi, Z., Knight, D.P., Huang, Y., Huang, L., Zhou, H., Shao, Z. and Chen, X. (2013) Insight into the Structure of Single Antheraea pernyi Silkworm Fibers Using Synchrotron FTIR Microspectroscopy. Biomacromolecules, 14, 1885-1892. http://dx.doi.org/10.1021/bm400267m

[26] Chen, L., Holman, H.-Y.N., Hao, Z., Bechtel, H.A., Martin, M.C., Wu, C. and Chu, S. (2012) Synchrotron Infrared Measurements of Protein Phosphorylation in Living Single PC12 Cells during Neuronal Differentiation. Analytical Chemistry, 84, 4118-4125. http://dx.doi.org/10.1021/ac300308x

[27] Yaji, T., Yamamoto, Y., Ohta, T. and Kimura, S. (2008) A New Beamline for Infrared Microscopy in the SR Center of Ritsumeikan University. Infrared Physics \& Technology, 51, 397-399. http://dx.doi.org/10.1016/j.infrared.2007.12.014

[28] Reches, M., Porat, Y. and Gazit, E. (2002) Amyloid Fibril Formation by Pentapeptide and Tetrapeptide Fragments of Human Calcitonin. The Journal of Biological Chemistry, 277, 35475-35480. http://dx.doi.org/10.1074/jbc.M206039200

[29] Tenidis, K., Waldner, M., Bernhagen, J., Fischle, W., Bergmann, M., Weber, M., Merkle, M.-L., Voelter, W., Brunner, H. and Kapurniotu, A. (2000) Identification of a Penta- and Hexapeptide of Islet Amyloid Polypeptide (IAPP) with 
Amyloidogenic and Cytotoxic Properties. Journal of Molecular Biology, 295, 1055-1071.

[30] Santi, S., Musi, V., Descrovi, E., Paeder, V., Di Francesco, J., Hvozdara, L., van der Wal, P., Lashuel, H.A., Pastore, A., Neier, R. and Herzig, H.P. (2013) Real-Time Amyloid Aggregation Monitoring with a Photonic Crystal-Based Approach. ChemPhysChem, 14, 3476-3482. http://dx.doi.org/10.1002/cphc.201300633 